\title{
Norm of units of quadratic fields.
}

\author{
By Yoshiomi Furuta
}

(Received Nov. 13, 1958)

Let $P$ be the rational number field and $\Omega=P(\sqrt{d})$ a real quadratic field, where $d$ is a positive square free integer, different from 2 . We denote by $\varepsilon_{0}$ a fundamental unit of $\Omega$; by $\varepsilon$ an arbitrary unit of $\Omega$; by $N$ the absolute norm; and by small Roman letters $a, b, \cdots, m, \cdots$ rational integers.

In this paper, we shall be concerned with the following problem:

"For what pair of integers $d, m$ does there exist in $\Omega$ a ring unit" $\varepsilon$ mod. $m$ with a negative norm: $N \varepsilon=-1$ ?"

Dirichlet gave some criteria on the question by means of power residue symbols. More recently it was investigated by A. Scholz, L. Rédei and others. In particular, Rédei [6], [7] etc. ${ }^{2}$, discussed it in detail by using the quadratic residue symbol and the fourth power residue symbol of Dirichlet, and finally Rédei [9] solved it completely as a problem related to the ideal class group of quadratic fields. On the other hand, Kuroda [5] and Furuta [1], [2] used the power residue symbol of Dirichlet and a generalized symbol to express the decomposition law of primes in some meta-abelian extensions, and also Tsunekawa [10] proved an interesting result concerning our problem. In the present paper, we shall give relationships between the norm of units of real quadratic fields and meta-abelian extensions, from which various results on our problem, in particular some of Rédei's results and Tsunekawa's theorem in a stronger from, can be deduced.

\section{$\S 1$. Restricted power residue symbol.}

Let $\Delta$ be an algebraic number field of finite degree, $\mathfrak{p}$ a prime ideal of $\Delta$ prime to 2 and $\alpha$ a number of $\Delta$, prime to $\mathfrak{p}$. Then for a non-negative rational integer $n$ the restricted $2^{n}$-th power residue symbol. $[\alpha / \mathfrak{p}]_{n}$ is defined as follows ${ }^{3)}$. For $n=0$ we set always $[\alpha / \mathfrak{p}]_{n}=1$. For $n \geqq 1[\alpha / \mathfrak{p}]_{n}$ is defined only when we have $[\alpha / \mathfrak{p}]_{n-1}=1$, and if this is really the case we set $[\alpha / \mathfrak{p}]_{n}$ $=(-1)^{x}$, where $\alpha^{\left(N p^{h}-1\right) / 2^{n}} \equiv(-1)^{x}$ (mod. $\mathfrak{p}$ ), $h$ being the smallest natural number with $2^{n} \mid N p^{h}-1$. For an ideal $\mathfrak{m}$ of $\Delta$ prime to both $\alpha$ and 2 with the

1) Namely, a unit $\varepsilon$ such that $\varepsilon$ is contained in the ring class mod. $m$.

2) See Rédei [9], in which the history and literatures of the subject is stated.

3) See Furuta [2]. If $\Delta$ containes all the $l$-th roots of unity for a fixed rational prime $l$, we shall have analogous results to this $\S 1$ by using $l$ instead of 2 . 
prime ideal decomposition $\mathfrak{m}=\mathfrak{p}_{1}{ }^{e_{1}} \ldots \mathfrak{p}_{t}{ }^{e_{t}}$ we set $[\alpha / \mathfrak{m}]_{n}=\left[\alpha / \mathfrak{p}_{1}\right]_{n}{ }^{e_{1}} \ldots\left[\alpha / \mathfrak{p}_{t}\right]_{n}{ }^{{ }} t$, when each $\left[\alpha / \mathfrak{p}_{i}\right]_{n}(i=1, \cdots, t)$ is defined. From the definition follows the following lemma in an analogous manner ${ }^{4}$ as in the case of the ordinary power residue symbol

Lemma 1. If $2^{n} \mid N \mathfrak{p}-1$ and $[\alpha / \mathfrak{p}]_{n}=1$, then $\left.\alpha \underset{[n]}{\equiv} 1(\bmod . \mathfrak{p})^{5}\right)$.

Furthermore, we can prove

LEMMA 2. If $2^{r} \| N \mathfrak{p}-1^{6)}$ and $[\alpha / \mathfrak{p}]_{r}=1$, then $[\alpha / p]_{n}=1$ for all $n$.

PROOF. For $n \leqq r$ we have trivially $[\alpha / p]_{n}=1$ by the definition. Let $n>r$ and $2^{n} \mid N \mathfrak{p}^{h}-1, h$ being as before. Let $\left(N p^{h}-1\right) / 2^{n}=k(N \mathfrak{p}-1) / 2^{r}$, where $k$ is an integer. Then, since $\alpha^{(N \mathfrak{p}-1) / 2^{r}} \equiv 1(\bmod . \mathfrak{p})$ by assumption, we have $\left.\boldsymbol{\alpha}^{(N \mathrm{p}}{ }^{h}-1\right) / 2^{n} \equiv \boldsymbol{\alpha}^{k(N \mathfrak{p}-1) / 2^{r}} \equiv 1$ (mod. p).

The next two lemmas follow immediately from Lemma 2 and the definition.

Lemma 3. If both $[\alpha / \mathfrak{p}]_{n}$ and $[\beta / p]_{n}$ are defined, then $[\alpha \beta / \mathfrak{p}]_{n}$ is also defined, and we have $[\alpha / \mathfrak{p}]_{n}[\beta / \mathfrak{p}]_{n}=[\alpha \beta / \mathfrak{p}]_{n}$.

Lemma 4. If $\left[\alpha^{k} / \mathfrak{p}\right]_{n}$ is defined for some odd rational integer $k$, then $[\alpha / p]_{n}$ is also defined, and we have $\left[\alpha^{k} / \mathfrak{p}\right]_{n}=[\alpha / \mathfrak{p}]_{n}{ }^{k}=[\alpha / \mathfrak{p}]_{n}$.

Lemma 5. For any prime ideal p prime to 2 and for any natural number $s$, the next two relations $\alpha \underset{[n]}{\equiv} 1$ (mod. $\mathfrak{p})$ and $\alpha \underset{[n, 1}{\equiv} 1$ (mod. $\left.\mathfrak{p}^{s}\right)$ are equivalent.

Proof. If $\alpha \underset{[n]}{\equiv} 1$ (mod. $\mathfrak{p}^{s}$ ), then trivially $\alpha \underset{[n]}{\equiv} 1$ (mod. $\mathfrak{p}$ ). Conversely suppose that $\alpha \underset{[n]}{\equiv}(\bmod \mathfrak{p})$, namely $\alpha=\beta^{2^{n}} \gamma, \gamma \equiv 1$ (mod. $\mathfrak{p}$ ) for some $\beta, \gamma \in \Delta$. Denoting by $S\left(\mathfrak{p}^{s}\right)$ the group of all $x \in \Delta$ such that $x \equiv 1\left(\bmod . p^{s}\right)$, we see that the order of the factor group $S(\mathfrak{p}) / S\left(\mathfrak{p}^{s}\right)$ is equal to $\varphi\left(\mathfrak{p}^{s}\right) / \varphi(\mathfrak{p})=p^{k}$ where $k=$ $f(s-1), N p=p^{f}$. Therefore $\gamma^{p^{k}} \equiv 1\left(\bmod . p^{s}\right)$, whence $\alpha^{p^{k}} \underset{[n]}{\equiv}\left(\bmod . p^{s}\right)$. Since $p$ is odd, we have $\alpha \underset{[n]}{\equiv} 1\left(\bmod . p^{s}\right)$.

Now we have the following

Lemma 6. We have $[\alpha / \mathfrak{p}]_{n}=1$ for all ${ }^{7} \quad n$ if and only if we have $\alpha^{k} \equiv 1$ (mod. $\left.\mathfrak{p}^{s}\right)$ for a natural number $s$ and for an odd rational integer $k_{\text {. }}$

Proof. From $\alpha^{k} \equiv 1\left(\bmod . p^{s}\right)$ follows $\alpha^{k} \equiv 1(\bmod . p)$, hence $\left[\alpha^{k} / \mathfrak{p}\right]_{n}=1$ for all $n$, and by Lemma 4 we have $[\alpha / \mathfrak{p}]_{n}=1$ for all $n$ since $k$ is odd. Conversely, suppose that $[\alpha / \mathfrak{p}]_{n}=1$ for all $n$. If $2^{r} \| N p-1$, then by Lemma 1 $\alpha \equiv 1\left(\bmod . p^{s}\right)$, i. e. $\alpha=\beta^{2^{r}} \gamma, \gamma \equiv 1\left(\bmod . p^{s}\right)$ for some $\beta, \gamma \in \Delta$. If we set $k=$ $\varphi\left(\mathfrak{p}^{s}\right) / 2^{r}$, then $k$ is odd and we see that $\beta^{k 2^{r}}=\beta^{\varphi\left(p^{s}\right)} \equiv 1\left(\bmod . \mathfrak{p}^{s}\right)$. Hence we

4) For instance, see Hasse, Bericht über neuere Untersuchungen und Probleme aus der Theorie der algebraischen Zahlkörper II (1926), p. 10.

5) $\alpha \underset{[n]}{\equiv}(\bmod . \mathfrak{p})$ means that $\alpha \equiv \beta^{2}$ (mod. p) for some $\beta \in \Delta$.

6) $2^{r} \| N_{\mathfrak{p}}-1$ means that $2^{r} \mid N_{\mathfrak{p}}-1$ and $2^{r+1} \nmid N_{\mathfrak{p}}-1$.

7) By Lemma 2 we may write " for $n$ such that $2^{n} \| N_{p}-1$ " instead of " for all $n$ ". 
have $\alpha^{k} \equiv 1\left(\bmod . p^{s}\right)$ for an odd $k$.

\section{$\S 2$. Norm of ring units of real quadratic fields.}

We denote hereafter by small Greek letters $\alpha, \beta, \cdots$ integers of the quadratic field $\Omega$, and by $\alpha^{\prime}, \beta^{\prime}, \cdots$ their conjugates with regard to $\Omega / P$. Let $d=q_{1} \cdots q_{t}$ be the prime number decomposition of $d$ in $P$, and $q_{1}, \cdots, q_{t}$ be all the prime divisors of $q_{1}, \cdots, q_{t}$ in $\Omega$ respectively. Further, assume hereafter that $m$ is odd.

LeMma 7. Let $m=\prod_{i=1}^{s} \mathfrak{p}_{i}{ }^{{ }_{i}} \prod_{j=1}^{t} \mathfrak{q}_{j}{ }^{{ }_{j}}$ be the prime ideal decomposition of $m$ in $\Omega$ where $\left(\mathfrak{p}_{i}, d\right)=1,1 \leqq e_{i}$ and $0 \leqq e_{j}$. Then $\alpha$ is contained in the ring class mod. $m$ of $\Omega$ if and only if

$$
\left\{\begin{array}{clc}
\alpha \equiv \alpha^{\prime} & \left(\bmod . \mathfrak{p}_{i}{ }^{\left.e_{i}\right)}\right. & i=1, \cdots, s, \\
\alpha \equiv \alpha^{\prime} & \left(\bmod . \mathfrak{q}_{j}{ }^{2 e^{j+1}}\right) & j=1, \cdots, t .
\end{array}\right.
$$

Proof. Since we have $\alpha-\alpha^{\prime}=b \sqrt{d}$ or $\alpha-\alpha^{\prime}=2 b \sqrt{d}$ with some $b$ according as $d \equiv 1$ (mod. 4) or $d \equiv 2,3$ (mod. 4), the lemma is clear.

THEOREM 1. In order that $N \varepsilon_{0}=1$ resp. -1 it is necessary and sufficient that $\left[\varepsilon_{0}{ }^{2} / q\right]_{n}=1$ resp. $\left[-\varepsilon_{0}{ }^{2} / q\right]_{n}=1$ for all $n^{7)}$ and for one of the prime divisors q prime to 2 of $d$.

PROOF. i) Since $\varepsilon_{0} \equiv \varepsilon_{0}{ }^{\prime}(\bmod . \sqrt{d})$, we have $N \varepsilon_{0} \equiv \varepsilon_{0}{ }^{2}$ (mod. $\sqrt{d}$ ), namely $\varepsilon_{0}^{2} \equiv 1$ or $-1(\bmod . \sqrt{d})$ according as $N \varepsilon_{0}=1$ or -1 . Hence, it follows from Lemma 6 that we have $\left[\varepsilon_{0}{ }^{2} / \mathfrak{q}\right]_{n}=1$ or $\left[-\varepsilon_{0}{ }^{2} / \mathfrak{q}\right]_{n}=1$ for all $\mathfrak{q}$ prime to 2 according as $N \varepsilon_{0}=1$ or -1 .

ii) Suppose that $\left[-\varepsilon_{0}{ }^{2} / \mathfrak{q}\right]_{n}=1$ for one of $\mathfrak{q} \mid d$ prime to 2 and for all $n$. Then by Lemma 6 we have $\varepsilon_{0}{ }^{2 k} \equiv-1$ (mod. $\left.\mathfrak{q}\right)$ for some odd $k$, hence $\left(N \varepsilon_{0}\right)^{k} \equiv$ -1 (mod. q), owing to $N \varepsilon_{0} \equiv \varepsilon_{0}{ }^{2}(\bmod \cdot \mathfrak{q})$. Since $k$ is odd, we have $N \varepsilon_{0} \equiv-1$ (mod. q), which means that $N \varepsilon_{0}=-1$, because $q$ is prime to 2 .

Now we prove the following ${ }^{8)}$

ThEOREM $2^{9)}$ In order that there exists in $\Omega$ a ring unit $\varepsilon$ mod. $m$ such that $N \varepsilon=-1$, it is necessary and sufficient that we have $\left[-\varepsilon_{0}{ }^{2} / q\right]_{n}=1$ for all $n^{7}$ and for one of the prime divisors $\mathfrak{q}$, prime to 2 , of $d$ and $\left[-\varepsilon_{0}{ }^{2} / \mathfrak{p}\right]_{n}=1$ for all $n^{7}$ ) and for all prime divisors $\mathfrak{p}$ of $m$.

8) Theorem 2 is a result stronger than that of Tsunekawa [10], i. e. we drop his assumption $N \varepsilon_{0}=-1$.

9) In the excluding cases where $d=2$ or $m$ is even, we can show easily the following facts: In case of $d=2$ we have $N \varepsilon_{0}=-1$. In case of $m$ being even, if $d \equiv 1$ (mod. 4) and $N \varepsilon_{0}=-1$ then $\varepsilon=\varepsilon_{0}{ }^{\varphi(2)}$ is a ring unit mod. 2 such that $N \varepsilon=-1$, where $\varphi$ is Euler's function in $\Omega$; if $2 \mid d$, then there is no ring unit $\varepsilon$ mod. 2 such that $N_{\varepsilon}=-1$; finally, if $d \neq 1(\bmod .4)$ and $2 \nmid d$, then always $N \varepsilon_{0}=-1$. 
Proof. $N \varepsilon=-1$ if and only if $N \varepsilon_{0}=-1$ and $\varepsilon=\varepsilon_{0}{ }^{k}$ for some odd $k$. On the other hand, by Lemma $7, \varepsilon$ is a ring unit mod. $m$ if and only if $N \varepsilon \equiv \varepsilon^{2}$ (mod. $\mathfrak{p}_{i}{ }^{{ }}{ }_{i}$ and $\left.\mathfrak{q}_{j}{ }^{{ }^{2}{ }^{+1+1}}\right)(i=1, \cdots, s ; j=1, \cdots, t)$. Hence it is necessary and sufficient for $\varepsilon$ to be a ring unit mod. $m$ that we have $N \varepsilon_{0}=-1$ and $\left(-\varepsilon_{0}^{2}\right)^{k} \equiv 1$ (mod. $\mathfrak{p}_{i}{ }^{{ }_{i}}$ and $\left.\mathfrak{q}_{j}{ }^{2 e_{j+1}}\right)$ for some odd $k(i=1, \cdots, s ; j=1, \cdots, t)$. The theorem follows immediately from Lemma 6 and Theorem 1.

\section{§3. Fields $\Omega\left(\sqrt{\varepsilon_{0}}\right)$.}

Lemma 8. We have $N \varepsilon_{0}=1$ if and only if $\Omega\left(\sqrt{\varepsilon_{0}}\right) / P$ is a non-cyclic extension of degree 4.

We have $N \varepsilon_{0}=-1$ if and only if $\Omega\left(\sqrt{\sqrt{d} \varepsilon_{0}}\right) / P$ is a cyclic extension of degree 4.

Proof. Let $\omega$ be equal to $\varepsilon_{0}$ or $\sqrt{d} \varepsilon_{0}$ according as $N \varepsilon_{0}=1$ or -1 , and put $K=\Omega(\sqrt{\omega})$. Let $\sigma$ and $\tau$ be the non-unit element of the Galois group of $\Omega / k$ and of $K / \Omega$ respectively, and let $U_{\sigma}$ be a representative of $\sigma$ in the Galois group of $K / P$. Since $\varepsilon_{0}{ }^{\sigma}=\varepsilon_{0}{ }^{-1}$ or $\left(\sqrt{d} \varepsilon_{0}\right)^{\sigma}=\sqrt{d} \varepsilon_{0} \cdot \varepsilon_{0}{ }^{-2}$ according as $N \varepsilon_{0}=1$ or $-1, K / P$ is a normal extension. On the other hand we have $\sqrt{\omega^{\tau}}=-\sqrt{\omega}, \sqrt{\omega}^{U_{\sigma}}=\sqrt{\omega} \gamma$ for some $r \in \Omega$, hence $\sqrt{\omega}^{U_{\sigma}^{z}}=\sqrt{\omega} N \gamma$. First, let $N \varepsilon_{0}=1$. Then $\omega=\varepsilon_{0},\left(\sqrt{\omega}^{U_{\sigma}}\right)^{2}=\varepsilon_{0}{ }^{\sigma}=\varepsilon_{0}{ }^{-1}$ and $\left(\sqrt{\omega} \bar{U}^{U_{\sigma}}\right)^{2}=(\sqrt{\omega} \gamma)=\varepsilon_{0} \cdot \gamma^{2}$. Therefore we have $\gamma= \pm \varepsilon_{0}, N \gamma=N \varepsilon_{0}=1$, hence $U_{\sigma}^{2}=1$, which means that $K / P$ is a non-cyclic extension. Next, let $N \varepsilon_{0}=-1$. Then $\omega=\sqrt{d} \varepsilon_{0},\left(\sqrt{\omega^{\sigma}}\right)^{2}=\left(\sqrt{d} \varepsilon_{0}\right)^{\sigma}$ $=\sqrt{d} \varepsilon_{0}{ }^{-1}$ and $\left(\sqrt{\omega}{ }^{U_{\sigma}}\right)^{2}=(\sqrt{\omega} \gamma)^{2}=\sqrt{d} \varepsilon_{0} \cdot \gamma^{2}$. Therefore we have $\gamma= \pm \varepsilon_{0}^{-1}, N \gamma$ $=N \varepsilon_{0}{ }^{-1}=-1$, hence $U_{\sigma}{ }^{2}=\tau$, which means that $K / P$ is a cyclic extension.

Now, for a while, suppose that $N \varepsilon_{0}=-1$. Let $d=q_{1} \cdots q_{t}$ be, as before, the prime number decomposition of $d$ in $P$. Then we see necessarily that $q_{i}=2$ or $q_{i} \equiv 1$ (mod. 4) $(i=1, \cdots, t)$. Let $K_{i}(i=1, \cdots, t)$ be a cyclic subfield of degree 4 of the $2^{n}$-th cyclotomic extensions over $P(n \geqq 4)$ or the cyclic subfield of degree 4 of the ray class field mod. $q$ over $P$ according as $q_{i}=2$ or not. Moreover, let $\chi_{i}$ be a generating character of the Galois group of $K_{i} / P$ $(i=1, \cdots, t)$. For $a \in P$ we put $\chi_{i}(a)=\chi_{i}\left(\left(\frac{K / P}{(a)}\right)\right)$ where $\left(\frac{K / P}{(a)}\right)$ is the Artin symbol. We set

$$
\chi=\chi_{1}{ }^{n_{1}} \cdots \chi_{t}{ }^{n_{t}}, n_{i}=1,3 \quad(i=1, \cdots, t) .
$$

Then $\Omega$ is the field corresponding to $\chi^{2}$. Denote by $A$ the field corresponding to $\chi$. Then all the divisors of $d$ and only these are completely ramified in $A / P$, and conversely a cyclic extension $A$ over $P$ of degree 4 with this property corresponds to a character $\chi$ defined by $(*)$.

In the rational number field the symbol $[a / p]_{n}$ is defined for $p=2$ as 
follows ${ }^{10)}:[a / 2]_{n}$ is defined only when $a \equiv 1$ (mod. $2^{n+1}$ ) and if this is really the case $[a / 2]_{n}$ is equal to 1 or -1 according as $a \equiv 1$ (mod. $2^{n+z}$ ) or not.

Now we have

THEOREM 3. If $N \varepsilon_{0}=-1$, then $\Omega\left(\sqrt{-1}, \sqrt{\varepsilon_{0}}\right) / P$ is a non-abelian extension, and, for some $\chi$ defined by (*) and for any rational prime $p$ with $p \equiv 1$ (mod. 4) and $(d / p)=1^{11)}$, we have

$$
\left(\varepsilon_{0} / \mathfrak{p}\right)=\chi(p)[d / p]_{2}
$$

where $\mathfrak{p}$ is a prime divisor of $p$ in $\Omega$, and $\left(\varepsilon_{0} / p\right)$ is the quadratic residue symbol in $\Omega(\sqrt{-1})$.

Proof. If we put $K=\Omega\left(\sqrt{\sqrt{d} \varepsilon_{0}}\right)$ and $K^{\prime}=\Omega\left(\sqrt{-\sqrt{d} \varepsilon_{0}}\right)$, then by Lemma $8 K$ and $K^{\prime}$ are both cyclic extension over $P$ of degree 4 , in which all divisors of $d$ are completely ramified. If we can show that at least in one of them only the divisors of $d$ are ramified, then by what we have remarked above we see that $\left(\sqrt{d} \varepsilon_{0} / \mathfrak{p}\right)=\chi(\mathfrak{p})$, and therefore $\left.{ }^{12}\right) \quad\left(\varepsilon_{0} / \mathfrak{p}\right)=\chi(p)[d / p]_{2}$, for some $\chi$ defined by $(*)$ and for any rational prime $p$ with $p \equiv 1$ (mod. 4$)$ and $(d / p)=1$. Hence to prove the theorem we have only to show that at least in one of $K$ and $K^{\prime}$ over $P$ only the divisors of $d$ are ramified. Since both in $K$ and in $K^{\prime}$ over $P$ only divisors of $2 d$ can be ramified, it remains only to prove that if $d$ is not even, then 2 is not ramified at least in one of $K$ or $K^{\prime}$ over $P$. Let $d$ be odd, and suppose that 2 is ramified in $K$. Denote by $A$, as before, the field corresponding to $\chi$, and $B$ the quadratic subfield of $A K$ over $\Omega$, distinct both from $A$ and from $K$. Then, since $A$ and $K$ are both cyclic over $P$ of degree $4, B$ is non-cyclic and biquadratic over $P$ and only the divisors of 2 are ramified. Hence we have $B=\Omega(\sqrt{a})$ where $a=-1$ or 2 . But $a=2$ does not occur, because otherwise we would have $A=\Omega\left(\sqrt{2 \sqrt{d} \varepsilon_{0}}\right)$, contrary to the fact that 2 is not ramified in $A / P$. Therefore we have $A=K^{\prime}$, and our assertion is proved.

Corollary. If we assume in Theorem 2 moreover that $\left(p / q_{i}\right)=1$ for all $q_{i} \mid d$, then we have

$$
\left(\varepsilon_{0} / \mathfrak{p}\right)=[p / d]_{2}[d / p]_{2} .
$$

ProOF. If $\left(p / q_{i}\right)=1$, then we have $\chi_{i}(p)=\left[p / q_{i}\right]_{2}$. Thus, our assertion follows from the theorem at once.

\section{§4. Applications.}

If Pell's equation $x^{2}-f y^{2}=-1$ is solvable, we call $f$ admissible. Suppose

10) cf. Furuta $[1$, p. 50].

11) $(d / p)$ is the quadratic residue symbol in $P$.

12) cf. Furuta [2, Lemma 1 and Lemma 2]. 
that $d(\neq 2)$ is squarefree and let $f=m^{2} d$. Then $f$ is admissible if and only if there exists in $P(\sqrt{d})$ a ring unit $\varepsilon$ mod. $m$ such that $N \varepsilon=-1$. By Theorem 2 and Corollary to Theorem 3 the following result is easily obtained:

a) Suppose that $d$ is admissible and that $m$ is divisible only by primes $p$ with $p \equiv 5$ (mod. 8) and $(p / q)=1$ for all $q \mid d$. Then $m^{2} d$ is addmissible if and only if we have $[p / d]_{2}[d / p]_{2}=-1$ for all $p \mid m$.

b ${ }^{13)}$ Let $d_{1}$ and $d_{2}$ be two positive odd integers and put $d=d_{1} d_{2}, \Omega_{1}=$ $P\left(\sqrt{d_{1}}\right), \Omega_{2}=P\left(\sqrt{d_{2}}\right), \Omega_{3}=P(\sqrt{d})$. Moreover, let $\varepsilon_{i}$ be a fundamental unit of $\Omega_{i}$ and suppose that $N \varepsilon_{i}=-1(i=1,2,3)$. Then $\sqrt{\varepsilon_{1} \varepsilon_{2} \varepsilon_{3}}$ is contained in $\Lambda=$ $P\left(\sqrt{-1}, \sqrt{q_{1}}, \cdots, \sqrt{q_{t}}\right)$ where $q_{1}, \cdots, q_{t}$ are all divisors of $d$.

$\mathrm{P}_{\mathrm{ROOF}}$. Let $p$ be any rational prime which decomposes completely in $\Lambda$, i. e., $(p / q)=1$ for all $q \mid d$, and $\mathfrak{P}, \mathfrak{p}_{1}, \mathfrak{p}_{2}, \mathfrak{p}_{3}$ be prime divisors of $p$ in $\Lambda, \Omega_{1}, \Omega_{2}$, $\Omega_{3}$ respectively. Then by Corollary to Theorem $3\left(\varepsilon_{1} \varepsilon_{2} \varepsilon_{3} / \mathfrak{P}\right)=\left(\varepsilon_{1} / \mathfrak{p}_{1}\right)\left(\varepsilon_{2} / \mathfrak{p}_{2}\right)\left(\varepsilon_{3} / \mathfrak{p}_{3}\right)$ $=\left[p / d_{1}\right]_{2}\left[d_{1} / p\right]_{2}\left[p / d_{2}\right]_{2}\left[d_{2} / p\right]_{2}[p / d]_{2}[d / p]_{2}=1$. Hence $p$ also decomposes completely in $\Lambda\left(\sqrt{\varepsilon_{1} \varepsilon_{2} \varepsilon_{3}}\right)$, i. e. $\sqrt{\varepsilon_{1} \varepsilon_{2} \varepsilon_{3}} \in \Lambda$.

c) ${ }^{14)}$ Let $d_{1}$ and $d_{2}$ be two admissible odd integers prime to each other, and put $d=d_{1} d_{2}$. If $\left[p / d_{2}\right]_{2}\left[d_{2} / p\right]_{2}=-1$ for one of the prime divisors $p$ of $d_{1}$, then $d$ is non-admissible.

Proof. Notations being as in b), we have $N \varepsilon_{1}=N \varepsilon_{2}=-1$ by the assumption for $d_{1}$ and $d_{2}$. We now assume that $N \varepsilon_{3}=-1$. Then by $\left.\mathbf{b}\right)$ the product $\varepsilon_{1} \varepsilon_{2} \varepsilon_{3}$ is a square number in $\Lambda$. Hence we have $\left(\varepsilon_{3} / \mathfrak{p}_{3}\right)=\left(\varepsilon_{1} / \mathfrak{p}_{1}\right)\left(\varepsilon_{2} / \mathfrak{p}_{2}\right)$ and by Theorem $1\left[-\varepsilon_{1}^{2} / \mathfrak{p}_{1}\right]_{2}=[-1 / p]_{2}\left(\varepsilon_{1} / \mathfrak{p}_{1}\right)=1$. Therefore, we see by Corollary to Theorem 3 that $\left(\varepsilon_{3} / \mathfrak{p}_{3}\right)=\left[-1 / \mathfrak{p}_{1}\right]_{2}\left(\varepsilon_{2} / \mathfrak{p}_{2}\right)=\left[-1 / \mathfrak{p}_{1}\right]_{2}\left[p / d_{2}\right]_{2}\left[d_{2} / p\right]_{2}$, whence $\left(\varepsilon_{3} / \mathfrak{p}_{3}\right)$ $=-\left[-1 / \mathfrak{p}_{1}\right]_{2}=-[-1 / p]_{2}$ by the assumption. On the other hand, we have $\left[-\varepsilon_{3}{ }^{2} / \mathfrak{p}_{3}\right]_{2}=[-1 / p]_{2}\left(\varepsilon_{3} / \mathfrak{p}_{3}\right)=1$ by Theorem 1, whence $\left(\varepsilon_{3} / \mathfrak{p}_{3}\right)=[-1 / p]_{2}$, which is a contradiction. Thus our assertion is proved.

Mathematical Institute, Nagoya University.

\section{References}

[1] Y. Furuta, A reciprocity law of the power residue symbol, J. Math. Soc. Japan, 10 (1958), 46-54.

[2] Y. Furuta, On meta-abelian fields of a certain type, Nagoya Math. J., 14 (1959), 193-199.

[3] T. Kubota, Über den bizyklischen biquadratischen Zahlkörper, Nagoya Math. J., 10 (1956), 65-85.

13) cf. Kuroda [4, Satz 11] and Kubota [3, Satz 1].

14) This criterion $\mathbf{c})$ is somewhat different from that of Rédei [7]. 
[4] S. Kuroda, Über den Dirichletschen Körper, J. Fac. Sci. Imp. Univ. Tokyo, Sec., I, 4 (1943), 383-406.

[5] S. Kuroda, Über die Zerlegung rationaler Primzahlen in gewissen nicht-abelschen galoischen Körpern. J. Math. Soc. Japan, 3 (1951), 148-156.

[6] L. Rédei, Über die Grundeinheit und die durch 8 teilbaren Invarianten der absoluten Klassengruppe im quadratischen Zahlkörper. J. Reine Angew. Math., 171 (1934), 131-148.

[7] L. Rédei, Über die Pellsche Gleichung $t^{2}-d u^{2}=-1$, J. Reine Angew. Math., 173 (1935), 193-211.

[8] L. Rédei, Bedingtes Artinsches Symbol mit Anwendung in der Klassenkörpertheorie, Acta Math. Acad. Sci. Hung., 4 (1953), 1-30.

[9] L. Rédei, Die 2-Ringklassengruppe des quadratischen Zahlkörpers und die Theorie den Pellschen Gleichung, ibid., 31-85.

[10] M. Tsunekawa, On the multiple solutions of $x^{2}-d y^{2}=-1$, Bull. Nagoya Inst. Technology, 8 (1956), 1-7 (Japanese). 